\title{
BULLEIIN OF THE
}

\section{AMERICAN MATHEMATICAL SOCIET Y}

\section{THE SIXTH SUMMER MEETING OF THE AMER- ICAN MATHEMATICAL SOCIETY.}

The Sixth Summer Meeting of the American MathematICAL Society was held at the Ohio State University, Columbus, Ohio, on Fridav and Saturday, August 25th and 26th, 1899. As has been the custom for several years, the meeting was held in conjunction with the American Association for the Advancement of Science. Aside from the material and incidental advantages, it has been found that the freedom of intercourse between the members of the Society and of Sections A and B of the Association, afforded by simultaneous meetings, serves to increase the interest in both gatherings, while the two-fold attachment of many members tends to enlarge the attendance. During the recent meeting of the American Association the initial steps were taken looking toward the still closer affiliation of the two societies for the purposes of the summer meeting, and the Committee of Section A was authorized to arrange for a joint meeting hereafter, if this should be deemed advisable.

The number of persons present during the meeting was over fifty, and included the following thirty-two members of the Society:

Professor W. W. Beman, Professor Henry Benner, Professor R. D. Bohannan, Professor C. H. Chandler, Professor L. E. Dickson, Professor C. L. Doolittle, Professor L. W. Dowling, Professor H. T. Eddy, Mr. H. B. Evans, Professor B. F. Finkel, Professor G. B. Halsted, Professor Thomas F. Holgate, Professor E. W. Hyde, Mr. H. G. Keppel, Professor T. E. McKinney, Professor A. MacFarlane, Professor W. H. Maltbie, Professor J. L. Markley, Professor Mansfield Merriman, Dr. G. A. Miller, Professor E. H. Moore, Professor A. B. Nelson, Professor H. B. Newson, Professor Alexander Pell, Professor G. T. Sellew, Professor J. B. Shaw, Dr. H. F. Stecker, Professor Ormond Stone, Dr. W. M. Strong, Professor A. L. P. Wernicke, Professor H. S. White, Professor R. S. Woodward. 
The first session opened at ten o'clock on Friday morning, and was presided over by Professor R. S. Woodward, President of the Society. On taking the chair the presiding officer congratulated the Society on the manifest interest in mathematical study and investigation in this country as indicated by the long list of papers to be presented, and contrasted the present rapid growth of mathematical knowledge with the conditions twenty or twenty-five years ago.

By holding extended sessions the full programme was completed by Saturday noon. On Friday evening nearly all the members in attendance dined together at the Chittenden Hotel. This informal dinuer has come to be a very interesting feature of the summer meeting and affords an opportunity for friendly interchange of thought upon mathematical topics that do not come up in the discussions of the regular programme.

At the Saturday morning session the Council announced the election of the following ten persons to membership in the Society: Professor Edgar Buckingham, Bryn Mawr College ; Dr. Warren G. Bullard, University of Vermont; Mr. Walter B. Ford, Albany, N. Y.; Miss Fanny C. Gates, Woman's College, Baltimore ; Miss Carrie Hammerslough, Columbia University ; Professor Thomas F. Nichols, Hamilton College ; Mr. Herbert W. Richmond, King's College, Cambridge, England; Professor George T. Sellew, Knox College, Galesburg, Illinois ; Professor E. B. Skinner, University of Wisconsin; Dr. George P. Starkweather, Yale University. Nine applications for membership were received.

The following papers were read :

(1) Dr. A. S. Chessin : "Note on relative motion."

(2) Mr. F. R. Moulton: "On surfaces of zero relative velocity and a certain class of special solutions in the problem of four bodies."

(3) Professor J. E. DAvies : "On the use of generalized differentiation in the solution of physical problems."

(4) Professor ARnold Emch : "A new class of link works."

(5) Mr. J. V. Collins: "A relation between point and vector analysis."

(6) Professor Florian Cajori : "John Speidell's 'New Logarithmes.' ',

(7) Mr. H. W. Richmond: "On analogues of the property of the orthocenter."

(8) Professor H. S. WHITE : "Irrational covariant conics of a plane cubic.", 
(9) Professor Frank Morley : "On the generalization of Desargues's theorem."

(10) Professor E. H. Mooke : "On certain crinkly curves."

(11) Dr. W. M. Strong:- "Note on non-quaternion number systems."

(12) Professor H. B. Newson : "On mixed groups."

(13) Dr. EDward Kasner : "The invariant theory of the inversion group."

(14) Miss E. N. Martin: "Note on the imprimitive substitution groups of degree fifteen, and on the primitive substitution groups of degree eighteen."

(15) Professor L. E. Dickson: "A new definition of the general abelian linear group."

(16) Professor L. E. Dickson : "Definition of various linear groups as groups of isomorphisms."

(17) Mr. W. B. Fite : "On the groups of cogredient isomorphisms that are abelian."

(18) Dr. G. A. MrLler : "On the groups that are direct products of two subgroups."

(19) Dr. G. H. Ling : "A proof that there are no simple groups of order $1440,1512,1680$, or $1800 . "$

(20) Dr. H. F. Stecker: "On a relation between the totality of the elliptic functions and a line complex."

(21) Dr. VIRgIL SNyder : "Geometric construction of the elliptic integral of the second kind, and of the Weierstrass sigma-function."

(22) Professor Alexander Pell : "Some applications of elliptic functions."

(23) Dr. L. T. Mone : "On Fresnel's wave surface."

Mr. Moulton's paper was offered to the Society through Professor Moore, Mr. Collins's through Professor Benner, Dr. Kasner's through Professor Fiske, Miss Martin's through Professor Harkness, and Mr. Fite's through Dr. Miller.

On account of the absence of the authors, Dr. Chessin's paper was read by Professor Woodward, Mr. Moulton's and Professor Morley's by Professor Moore, Professor Davies's by Professor Hyde, Professor Emch's by Professor Newson, Professor Cajori's by the Secretary, Miss Martin's, Dr. Ling's and Dr. Snyder's by Dr. Miller, and Dr. More's by Professor Brace of the University of Nebraska. The papers by Mr. Richmond, Dr. Kasner, and Professor Dickson (No. 15) were read by title. Abstracts of the papers are given below.

Considering space motion in the theory of relative motion as a perturbation of the motion which the given system 
would have if space motion did not exist, we arrive at the notion of a "perturbative function of relative motion." This function is the sum of three homogenous functions of $p, q, r$ (components of the angular velocity $\omega$ of space rotation) of the respective degrees 0,1 , and 2 . The purpose of Dr. Chessin's paper is to give the mechanical significance of the third of these three functions. The author shows that it is always possible to consider $\omega$ as the diagonal of a parallelogram constructed on two angular velocities $\omega_{1}$ and $\omega_{2}$, the direction of $\omega_{1}$ being one about which relative rotation is possible, whereas such a rotation is impossible about the direction of $\omega_{2}$. In this connection it was remarked that it is sometimes useful to express the kinetic energy of rotation of a body referred not to its principal axes of inertia, but to a system of conjugate axes. If then $G$ denote the kinetic energy of space rotation,

$$
2 G=A^{\prime} \omega_{x}^{2}+B^{\prime} \omega_{y}{ }^{2}+C^{\prime} \omega_{z}^{2},
$$

where $\omega_{x}, \omega_{y}, \omega_{z}$ are the components of $\omega$ along and $A^{\prime}, B^{\prime}$, $C^{\prime}$ the moments of inertia about the conjugate (generally oblique) axes $X Y Z$ of the ellipsoid of inertia. Now, if we take for $X$ the direction of $\omega_{1}$, then

$$
\begin{gathered}
G=G_{1}+G_{2}, \\
G_{1}=\frac{1}{2} A^{\prime} \omega_{1}^{2}, \quad G_{2}=\frac{1}{2} I \omega_{2}^{2},
\end{gathered}
$$

$I$ denoting the moment of inertia about an axis in the plane conjugate with the direction of $\omega_{1}$. Likewise, if we take for $X$ the direction of $\omega_{2}$, then

$$
G_{2}=\frac{1}{2} A^{\prime} \omega_{2}^{2}, \quad G_{1}=\frac{1}{2} I \omega_{1}^{2} .
$$

$G_{2}$ is exactly the third of the three terms of which $\omega$ is composed. Hence its mechanical significance.

Mr. Moulton's paper discussed the motions of four bodies, three of finite masses and one infinitesimal. The finite bodies were supposed to move according to one or other of the Lagrangian solutions, in circles around their common center of gravity. It was shown that the surfaces of zero relative motion separate those portions of space in which the infinitesimal body may move from those in which it cannot move. There are just twenty-eight solutions for which the system remains of constant form and size, and revolves in an arbitrary period. 
The purpose of Professor Davies's paper was to call attention to some of the earlier investigations on the subject of generalized differentiation, and in particular to an apparently little known paper by Buchwaldt, a captain in the Danish army, who critically reviewed the work of Liouville, previously published in the Journal de l'École Polytechnique, for 1832 , et seq. In view of some of the recent papers of Oliver Heaviside, wherein the usefulness of generalized differentiation in problems in electricity is pointed out, it was shown that attention had been called, long ago, by Liouville to the great value of this process in the solution of physical problems generally and that it is likewise valuable in analysis for certain inverse problems.

Professor Emch's paper, which is intended for publication in the Annals of Mathematics, is in abstract as follows :The element of the linkwork is the cell $O A_{1} B_{1} A_{2}$ -

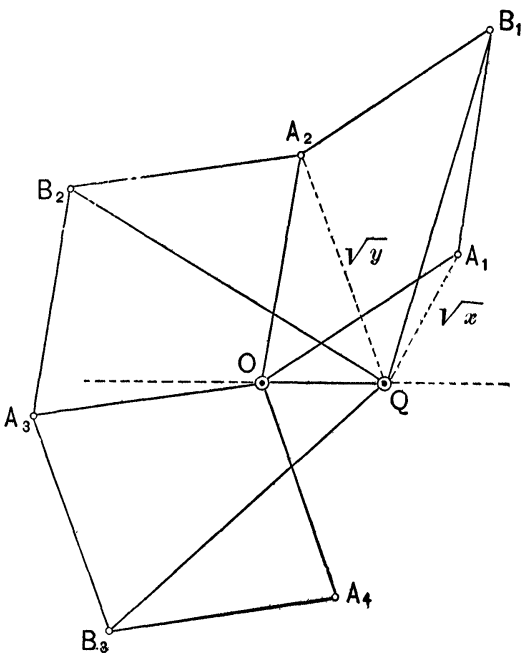
$O \cdot Q B_{1}$ in which $O, Q$ are fixed points, and $O A_{1}=A_{1} B_{1}$ $=B_{1} A_{2}=A_{2} O$. Putting $Q A_{1}=\sqrt{ } \bar{x}, Q A_{2}=\sqrt{ } \bar{y}, Q B_{1}=R$, $O A_{1}=r, O Q=c$, and $(R+r)^{2}=\alpha,(r+c)^{2}=\beta,(r-c)^{2}$ $=\gamma,(R-r)^{2}=\delta$, the relation exists

$$
\begin{aligned}
& \int^{y} \frac{d y}{\sqrt{(y-\alpha)(y-\beta)(y-\gamma)(y-\delta)}} \\
& -\int^{\delta} \frac{d x}{\sqrt{(x-\alpha)(x-\beta)(x-\gamma)(x-\delta)}}=a
\end{aligned}
$$

where $a$ is a constant. Let $v$ and $u$ designate the values of these integrals, so that $v-u=a$. The variables $x$ and $y$ may be expressed by elliptic functions

$$
x=\lambda(u), \quad y=\lambda(v) \text {. }
$$

A second cell may be added having the same fixed points $O$ and $Q$ and the link $\mathrm{OA}_{2}$ in common with the first cell. In 
the same manner any number of cells may be added, thus forming a link-work of one degree of freedom. If the linkwork consists of $n$ cells, $\overline{O A_{n+1}}$ will be the last link and the argument belonging to $\overline{Q A_{n+1}}$ will be $u+n \cdot a$. Supposing $w_{1}$ and $w_{2}$ as the periods of $\lambda(u)$, the quantities $R, r$, and $c$ may be chosen in such a manner that

$$
a=\frac{m_{1} w_{1}+m_{2} w_{2}}{n}
$$

where $m_{1}$ and $m_{2}$ designate integers. In this case the linkwork closes, $i$. e., the point $A_{n+1}$ will coincide with $A_{1}$, and the condition of closing is independent of the value of $u$, or of the original position of the link-work. In the special cases of closed link-works of three and four cells the condition reduces to $R=r$ and $R^{2}-r^{2}=r^{2}-c^{2}$, respectively. A certain transformation of the link-work leads to Jacobi's construction of elliptic functions and Poncelet's poristic polygons.

In his Quaternions (second edition, page 22) Tait asserts that "The theory of 'geometrical nets' is very fully developed in Hamilton's Elements, and the method is shown to include as particular cases the processes of Grassmann's Ausdehnungslehre and Möbius's Barycentrische Calcul."' This passage is criticized by Mr. Collins on two grounds: first, that it is too sweeping; and secondly, that if point and vector analysis are so closely connected, this relationship calls for a careful examination. The question arises, then, how can the two be brought together in a harmonious system. In his Elements, instead of studying point-vector analysis in its simplest form, Hamilton makes out of the simple expressions of point analysis very complex ones, anharmonic ratios in fact. In this way he limits the application of this analysis to the so-called modern geometry.

By Hamilton's $\rho$ and Grassmann's $p$ is or should be meant a complex concept consisting of a point $p$ held in position by a vector from an origin $\rho$. Here $p$ we will say denotes an infinitesimal portion of a line and $p \rho$ denotes $p$ at the end of $\rho$ whose initial point is at the origin. Thus $p \rho$ standing now for both Hamilton's $\rho$ and Grassmann's $p$ is really complex, just as $\varepsilon(=T \varepsilon U \varepsilon$ or $S \varepsilon+V \varepsilon)$ is complex in quaternions, or as $p_{1} \varepsilon_{1} \varepsilon_{2}$ is complex in the Ausdehnungslehre. Using this notation the fundamental equations of point analysis become

$$
m_{1} p \rho_{1}+m_{2} p \rho_{2}=\left(m_{1}+m_{2}\right) p\left(\frac{m_{1} \rho_{1}+m_{2} \rho_{2}}{m_{1}+m_{2}}\right),
$$




$$
\begin{aligned}
& \Sigma m p \rho=\Sigma m \cdot p\left(\frac{\sum m \rho}{\sum m}\right) \\
& p \rho_{2}-p \rho_{1}=0 p\left(\frac{\rho_{2}-\rho_{1}}{0}\right) .
\end{aligned}
$$

Hyde in his Directional Calculus writes these equations in duplicate, $i$. e., first in the simple point analysis form, and then in the vector analysis form, the vectors being indicated as the difference of units points. If the above notation and equations he used in the solution of problems, the point equations and vector equations correspond and agree at each step of the solution. If $p$ be divided out of any equation, a vector equation is left, while if the $p$ 's be given the subscripts of the $\rho$ 's, and the $\rho$ 's be removed, we have the regular point analysis equations. Thus this notation throws light on any solution or equation from two directions. The vector equations have the advantage that the origin can be taken anywhere; the point equations that they are always symmetrical. Because the fundamental concept dealt' with in point analysis is complex, the notation used to present it should be complex until the subject is well grasped.

Professor Cajori remarked in his paper that no general history of mathematics published in England or in Europe makes mention of John Speidell, though it is quite generally known to English readers through certain special articles, for example, J. W. L. Glaisher's report on "Tables" in the British Association Report for 1873, that Speidell is the author of the earliest table of natural logarithms. The book appeared in 1619, only five years after Napier's famous work. A fac-simile of the title-page of the edition of 1622 was exhibited to the Society.

Mr. Richmond's memoir deals first with the systems of $2 n$ points in a space of $n$ dimensions $S_{n}$, such that the $S_{n-1}$ determined by any $n$ points is perpendicular to that determined by the remaining $n$ points. Here for 'perpendicular' we may substitute 'conjugate as to a fixed quadric,' the absolute. More generally in $S_{n}$ let there be $p$ points $u_{1}=0, \cdots, u_{p}=0$ and let the absolute be

$$
\sum_{1}^{p} k_{r} u_{r}^{2}=0
$$

then any two spaces $S_{n-1}$ which contain all the $p$ points are necessarily perpendicular. For a proper quadric the values 
of $p$ range from $n+1$ to $2 n$; for the euclidean absolute (passing over the case of points all at infinity) $p$ ranges from $n+2$ to $2 n$. Such a system of $p$ points is called an orthogenic system. The system of $n+1$ orthogenic points is the self-conjugate pyramid; accordingly the system of $u+2$ points is selected for special consideration. This is the 'polviereck' in the case of the conic $(n=2)$, and the 'polfünfeck' in that of the quadric in 3 dimensions. In the euclidean case, it is pointed out, inter alia, that the centroids of all pairs formed from the $n+2$ points lie on a sphere; and if further $n$ be even, it is remarked that $n+1$ points determine uniquely $\frac{1}{2} n$ others, the whole forming an orthogenic system.

Professor White's paper embodied the results of some recent investigations upon a plane cubic curve and the systems of conics connected with it. and will be published in the Transactions of the American Mathematical Society. Two doubly infinite systems of conics are known (Hilbert in Liouville, 1888) which are related to the general plane cubic by an invariant property, namely, their equations are reproduced (to a factor) by an operation which transforms the cubic into its Hessian. Professor White finds each of these systems of conics to be the system of conic polars to a new cubic curve, and (1) these two cubics are coördinate with the fundamental cubic by virtue of a cyclic relationeach gives rise to the other two in the same way as the first did to the second and third ; (2) all three lie in the syzygetic sheaf containing the first and its Hessian ; (3) finally, the set of three are characterized by having their Cayleyan (curve of third class) in common. Two class-cubics defined by the self-reproductive property with respect to the transverse of this same operation prove to be coördinate to the conjugate class-cubic of the fundamental, and to be themselves conjugate to the two above described in the sense of Aronhold (Crelle, 55). They have the Cayleyan for their common Hessian. From these results, using the equation of the conjugate class-cubic, the covariant equations are written for conic polars of four cubics related to the Hessian just as the first four are to the Cayleyan.

Professor Morley communicated a proof of the theorem that all full spreads of order $n$ in a linear space $S_{m}$ which pass through

$$
\frac{(m+n-1) !}{m !(n-1) !}+1
$$


given points are apolar with a determinate full spread of class $n$ in an intersecting space $S_{m-1}$. This paper is intended for publication in the Transactions.

Professor Moore's paper treats (1) of the area filling curves $x=\varphi(t), y=\psi^{\prime}(t)$ of Peano and Hilbert, and in particular (2) of the curve $y=\psi(t)$, connected with the Peano curve, which is continuous and has at no point a progressive or a regressive non-vertical tangent, and at no point a progressive and a regressive vertical tangent. This curve from the simplicity of its geometric definition and from the intuitive clearness of its properties appears to be fit to replace the classical Weierstrass curve as the standard example of continuous curves having no tangents. The paper will be published in the Transactions.

Dr. Strong announced the following theorem : Any degenerate uon-quaternion number system whose multiplication table contains $m$ independent products can be reduced to a form such that the independent products will be $l_{1}, l_{2}, l_{3}, \cdots, l_{m}$, and will occur in the order here given. No independent product will be the product of units which themselves enter products. By 'degenerate system' is meant a system from which the modulus has been deleted; the term 'independent product' denotes a product of two units which is linearly independent of the products preceding it; and the 'order' of products in the multiplication table of nonquaternion number systems is defined thus: $l_{i} \cdot l_{i+a}$ and $l_{i+a} \cdot l_{i}$ precede $l_{i+b} l_{i+c}$ where $b, c \neq 0 ; l_{i} \cdot l_{i+a}$ and $l_{i+a} \cdot l_{i}$ precede $l_{i} \cdot l_{i+b}$ and $l_{i+b} \cdot l_{i}$ if $a<b ; l_{i+a} l_{i}$ precedes $l_{i} l_{i+a}$ where $a \neq 0$.

The object of Professor Newson's paper was to exhibit a method for treating mixed groups of projective transformations (or collineations). The method was applied to one mixed group as an example. Mixed groups contain two distinct sets of transformations, one set forms a continuous group and the other set forms a multitude of discontinuous groups. All projective transformations of the plane which leave a pair of points $A$ and $B$ separately invariant form a continuous group $G(A B)$; add to these all transformations which interchange $A$ and $B$, and we have the mixed group $m G(A B)$. All real projective transformations which interchange $A$ and $B$ were determined synthetically; they fall into four distinct classes. Those of the first class are involutoric, $i$. e., of period two; those of the other three classes are non-periodic and are designated as elliptic, para- 
bolic, and hyperbolic, respectively. The paper will be published in the Kansas University Quarterly.

To every group of transformations corresponds a geometry, or theory of invariants (Klein, Lie). The group considered by Dr. Kasner is the six-parameter group generated by the inversions of the plane; the corresponding inversion geometry is formally identical with projective geometry on a quadric surface. Two principal methods are developed for studying the invariant properties of geometric configuration. $1^{\circ}$ Quaternary.-A curve whose equation in tetracyclic coördinates $x_{i}$ connected by the quadratic identity $Q=0$ is $f_{n}=0$ is also represented by $f_{n}+M Q=0$, where $M$ is any form of order $n-2$; but of the entire system of forms $f_{n}+M Q$, there is one and only one $(F)$ which is apolar to $Q$. The final result may be stated : the (quaternary) inversion concomitants of the curve are the projective concomitants of $F, Q . \quad 2^{\circ}$ Double binary.-A point being defined by the parameters $\lambda_{1}: \lambda_{2}, \mu_{1}: \mu_{2}$ of the pair of minimal lines passing through it, the equation of the curve to be studied takes the form $\varphi\left(\lambda_{1}{ }^{m} \lambda_{2}, \mu_{1}{ }^{n} \mu_{2}\right)=0$; the (binary) inversion concomitants of the curve are the concomitants of the double binary form $\varphi$, with respect to independent linear transformations of $\lambda$ and $\mu$. The relations between $1^{\circ}$ and $2^{\circ}$ were considered in detail. It was shown for example that the binary and quarternary polar theories coincide; and a series of methods was given of which $2^{\circ}$ is the first member. Application was made to circles and cyclic curves.

In Miss Martin's paper it was shown that there are 68 imprimitive groups of degree fifteen. Of these 28 have their elements divisible into three systems of imprimitivity, 27 into five systems, while the remaining 13 have their elements divisible in both ways. In addition to the symmetric and to the alternating groups of degree eighteen only two other primitive groups have been found. One is a triply transitive group of order 18.17.16; the other is a doubly transitive simple group of order 16. 17.8 contained selfconjugately in the former.

Professor Dickson's papers are intended for publication in the Transactions. An abstract giving some of the results of both papers was published in the Bulletin, vol. 5 , p. 331. Mr. Dickson also presented a preliminary communication containing an elementary proof of a theorem by Professor Moore on the abstract form of the symmetric group on $k$ letters. 
Mr. Fite's paper presented the following considerations : The group of cogredient isomorphisms of any group $G$ cannot be the direct product of cyclical groups whose orders are such that one of them is not a divisor of the least common multiple of the others. If the group of cogredient isomorphisms is abelian, the order of every one of its operators is the order of a commutator of $G$. If $G$ is of order $p^{m}$ and contains an abelian subgroup of order $p^{m-a}$, its group of cogredient isomorphisms contains no operator of order greater than $p^{a}$, if it is abelian ; if the largest abelian subgroup of $G$ is of order $p^{m-a}$, where $\alpha+\frac{1}{2}(m-1), G$ cannot have an abelian group of cogredient isomorphisms of order $p^{m-1}$. If $G$ is of order $p^{m}$ and contains an abelian subgroup of order $p^{m-1}$, it cannot have an abelian group of cogredient isomorphisms of order $p^{m-a}$, where $\alpha<\frac{1}{2}(m-1)$.

Dr. Miller's paper, which will be offered for publication to the Transactions, is in abstract as follows:

If a group $G$ contains two self-conjugate subgroups $G_{1}, G_{2}$ which have just $K$ common operators, and if we form all the possible commutators $s^{-1} t^{-1} s t$ by taking $s$ from $G_{1}$ and $t$ from $G_{2}$ (or vice versa) then these commutators generate a self-conjugate subgroup of $G$ which is contained in $K$, and each operator of one of the two subgroups $G_{1}, G_{2}$ transforms every operator of the other into itself multiplied by some one of the given commutators. If $G$ is generated by $G_{1}$ and $G_{2}$, and if $K=1$, then $G$ is said to be the direct product of $G_{1}, G_{2}$. Two theorems are proposed:

Theorem I. If the order $g$ of a group $G$ is divisible by $p^{a}$ but not by $p^{a+1}, p$ being any prime number, and if all the conjugates of any operator $(s)$ contained in a subgroup $P$ of order $p^{a}$ may be obtained by transforming $s$ by means of operators of $P$, then $G$ is the direct product of $P$ and some other subgroup of $G$, and it contains a selfconjugate subgroup of each of the orders $g \div p^{\beta}(\beta=0,1,2$, ...., $\alpha-1$ ).

Theorem II. If the order of a group is $m n$ ( $m$ being prime to $n$ ) and if it contains a subgroup of order $m$ which has the property that each of its operators is transformed by some one of the operators of this subgroup in the same way as it is transformed by any given operator of the entire group, then the group is the direct product of two subgroups whose orders are $m$ and $n$ respectively.

Hölder, Cole, and Burnside have examined all the orders up to 1093 in regard to possible simple groups and proved 
that there are no simple groups of any of these orders with exception of those already known whose orders are 60, 168, 360, 504, 660 and 1092. At the February meeting of this Society Dr. Ling presented a paper in which all the other orders that do not exceed 2000 , except four, were examined. The orders 1440, 1512, 1680 and 1800 presented difficulties which could not then be overcome. With the assistance of Dr. Miller, Dr. Ling has now examined these orders and the result reached is that there is no simple group whose order lies between the numbers 1092 and 2001. These results will be incorporated in the paper previously presented to the Society and will be offered to the American Journal of Mathematics for publication.

The fundamental considerations in Dr. Stecker's paper were these : (1) the six line coördinates of a complex satisfy the identical relation $p_{1} p_{2}+p_{3} p_{4}+p_{5} p_{6}=0$, and conversely, any six quantities satisfying such an identical relation may be treated as line coördinates of a complex; (2) the biquadratic which enters into an elliptic integral has three irrational invariants each of which is the product of two invariant determinants. These three irrational invariants, $A, B, C$, satisfy the relation $A+B+C=0$. Hence the above-mentioned six determinants may be treated as line coördinates of a complex. Several interesting results were pointed out.

Dr. Snyder's paper describes the models of the functions $\sigma(u)$ and $\zeta(u)$, recently, prepared by the author under the direction of Professor Klein. These models, which are to be reproduced in plaster, are constructed with the same constants $\left(g_{2}=0, g_{3}=4\right)$ employed by Professor Burkhardt in the case of $\wp^{\prime}(u)$ and $\gamma^{\prime}(u)$, thus completing the set to a certain extent.

Professor Pell's paper dealt with the curves on an ellipsoid, called by E. Cosserat the ' $D$ ' lines. The author expressed the coördinates of these lines for the ellipsoid as uniform functions of one parameter ; by means of these expressions he traced the path of the curves, and deduced some interesting properties of these lines. The paper will be offered for publication to the Transactions.

Dr. More pointed out in his paper that it is generally assumed that unless a ray of light in a biaxial crystal is refracted along an optic axis, it will break up into two raysthe ordinary and the extraordinary - but the fact is that there are an infinite number of directions along which the ray is not 
divided. Suppose the section of Fresnel's wave surface by a coördinate plane to consist of a circle and an ellipse and let ( $A$ be the center of a wave disturbance in the surface of a crystal. Draw any radius vector $O B C$, cutting the circle at $B$ and the ellipse at $C$; the intersection of tangents at $B$ and $C$ will be $S$, a point on the tangent plane at the point of incidence. Then $S B$ and $S C$ will be the wave fronts of an ordinary and an extraordinary ray. Since these two lines are tangents at the points of intersection of a single radius vector $O B C$, the latter is the path of both an ordinary and an extraordinary ray. Therefore from the indices of refraction we may compute an incident ray which will not be divided but will move along $O B C$, and since there are an infinite number of points $S$ there are an infinite number of directions for which the ray is not divided. The locus of the point $S$ is a curve of the eighth degree and may be expressed in a simple and symmetrical form. By a simple transformation of the equation uniaxial crystals are included in this treatment.

Thomas F. Holgate, Acting Secretary.

Evanston, Illinors.

\section{REPORT ON THE RECENT PROGRESS IN THE THEORY OF LINEAR GROUPS.}

BY PROFESSOR L. E. DICKSON.

(Read before Section A of the American Association for the Advancement of Science, Columbus, Ohio, August 22, 1899.)

THIs report is intended to supplement in certain directions. the report* of Dr. Miller on group theory read at the last summer meeting of the American Association. In order to allow space for important details, I will consider neither continuous nor discontinuous linear groups of infinite order, but confine my report to finite linear groups. Of these I shall consider first the finite collineation groups and afterwards the linear congruence groups and the more general linear groups in Galois fields.

Part I.-Finite groups of linear substitutions.

\$1. General Theorems on Finite Linear Groups.

The following theorem was stated by Jordan in 1878 and proven in various ways by Lipschitz, Kronecker, Rost,

* Bulletin, Feb., 1899, p. 227. 